\title{
Synthesis of Water-Soluble Vinyl Selenides and Their High Glutathione Peroxidase (GPx)-Like Antioxidant Activity
}

\author{
Samuel Braverman, ${ }^{* a}$ Marina Cherkinsky, ${ }^{a}$ Yulia Kalendar, ${ }^{a}$ Ranjan Jana, ${ }^{a}$ Milon Sprecher, ${ }^{a}$ Israel Goldberg ${ }^{b}$ \\ a Department of Chemistry, Bar-Ilan University, Ramat-Gan 52900, Israel \\ Fax+972(3)7384053; E-mail: Shmuel.Braverman@biu.ac.il \\ b School of Chemistry, Tel-Aviv University, Ramat-Aviv 69978, Israel \\ Received: 25.08.2013; Accepted after revision: 10.10.2013
}

\begin{abstract}
A convenient procedure for the synthesis of novel bis-(1hydroxymethyl-2-halo-3-hydroxy-1-propylene) selenides has been developed. On oxidation these compounds form novel seleno-spiro compounds and their glutathione peroxidase mimetic activity has been studied. They promote the hydrogen peroxide oxidation of phenylmethanethiol to the corresponding disulfide via a catalytic cycle.
\end{abstract}

Key words: alkynes, selenium, oxidation, spiro compounds, diols

Glutathione peroxidase (GPx) is a selenoenzyme that protects cells by catalyzing the reduction of peroxides with the stoichiometric reductant glutathione. ${ }^{1}$ The enzyme catalytic site includes a selenocysteine residue in which the selenium undergoes a redox cycle involving the selenolate anion as the active form, which reduces hydroperoxides. The selenol is first oxidized to a selenenic acid EnzSeOH, which reacts with reduced glutathione GSH to form the selenyl sulfide EnzSeSG. A second glutathione then regenerates the active form of the enzyme by attacking the EnzSeSG to form the oxidized glutathione GSSG. The overall catalytic cycle is depicted in Figure $1 .{ }^{2}$

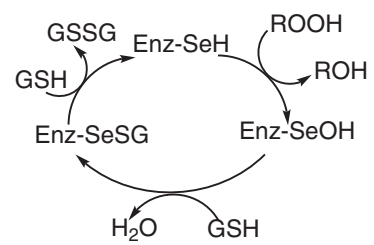

Figure 1 Catalytic cycle for the reduction of peroxides

Ebselen [2-phenyl-1,2-benzoisoselenazol-3(2H)-one] has been known as a mimetic of glutathione peroxidase (GPx), able to interact with active oxygen species present in living cells. ${ }^{3-6}$ However, its use under nonenzymic conditions as a catalyst for hydrogen peroxide oxidation of thiols to disulfides gave poor results. Recently, Back et al. ${ }^{7,8}$ have demonstrated the exceptional glutathione peroxidase-like activity of the simple bis(3-hydroxypropyl) selenide and the unexpected role of its oxidation product, spirodioxaselenanone, as an intermediate in the catalytic redox cycle for GPx.

SYNTHESIS 2014, 46, 0119-0125

Advanced online publication: 13.11.2013

DOI: 10.1055/s-0033-1338555; Art ID: SS-2013-Z0588-OP

(c) Georg Thieme Verlag Stuttgart · New York
In view of the known role of organoselenium compounds in biological systems, ${ }^{9-12}$ in addition to their unique characteristics in organic synthesis, ${ }^{13}$ the design and synthesis of novel organoselenium compounds with potential biological activity constitutes an ongoing challenge. Recently, we have found that in situ prepared selenium dichloride, readily obtained from elemental selenium and sulfuryl chloride, ${ }^{14}$ undergoes smooth 1,2-addition to the triple bond of various propargylic alcohols resulting in the formation of symmetrical (Z,Z)-bis(1-hydroxymethyl-2chlorovinyl) selenides $\mathbf{1}$ in high yields and in completely regio- and stereospecific manner (Scheme 1). ${ }^{15,16}$ Of special mechanistic interest is the observed syn-addition and anti-Markovnikov orientation.

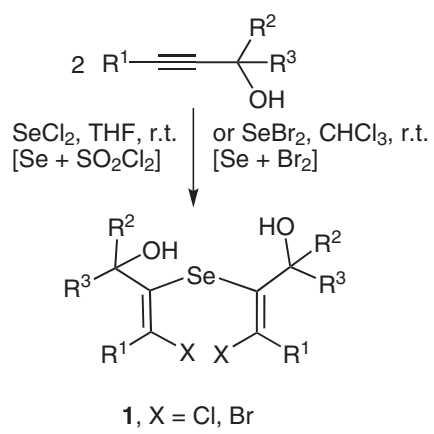

Scheme 1 Regio- and stereospecific synthesis of functionalized divinyl selenides

Inspired by the above findings, we applied our regio- and stereospecific $\mathrm{SeCl}_{2}$ addition protocol in the planned preparation of novel GPx-mimetics of spirodioxaselenurane type. ${ }^{8}$ Those divinyl selenides formed by syn-addition of $\mathrm{SeCl}_{2}$ to the triple bond, which do not bear a vicinal hydrogen capable of $c$ is elimination, gave stable selenoxides $\mathbf{2 a}, \mathbf{b}$ and selenone $\mathbf{3}$ in good yield upon oxidation with tert-butyl hydroperoxide or with 2 equivalents of MCPBA, respectively (Scheme 2). The divinyl selenoxides $\mathbf{2 c}, \mathbf{d}$ derived from the unsubstituted or monosubstituted propargyl alcohols are relatively unstable, presumably due to syn-elimination. The divinyl selenoxides $\mathbf{2 a}$ and $\mathbf{2 b}$ do not cyclize to spiro compounds (see below) presumably because of ring strain of four-membered rings. 


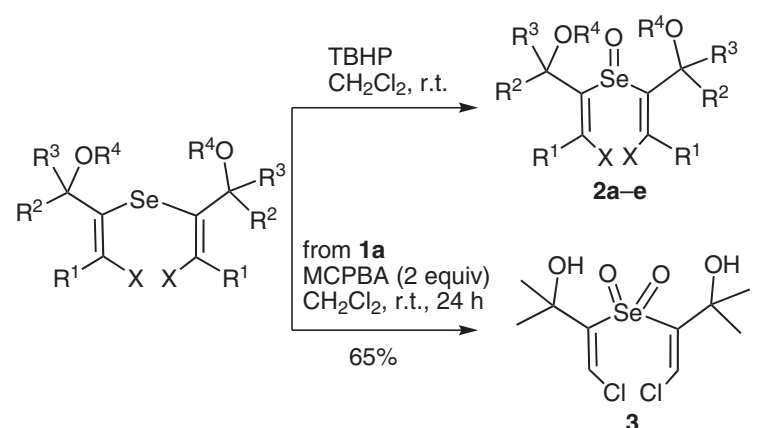

2a $R^{1}=R^{4}=H, R^{2}=R^{3}=M e, X=C l ; 88 \%$

2b $R^{1}=R^{4}=H, R^{2}=R^{3}=M e, X=B r ; 87 \%$

2c $R^{1}=M e, R^{2}=R^{3}=R^{4}=H, X=C l ; 85 \%$

2d $R^{1}=R^{2}=R^{3}=R^{4}=H, X=C l ; 82 \%$

2e $R^{1}=R^{2}=R^{3}=H, R^{4}=B n, X=C l, 93 \%$

Scheme 2 Oxidation of divinyl selenides

In contrast to the above, were our findings in the reaction of selenium dihalides with homopropargyl alcohol. ${ }^{16} \mathrm{Al}-$ though some loss of stereo- and regioselectivity was observed, Z-anti-Markovnikov adducts $\mathbf{4}$ were chromatographically isolated as major products and underwent oxidation with $\mathrm{H}_{2} \mathrm{O}_{2}$ to the corresponding fivemembered spiroselenuranes $\mathbf{5}$ with exocyclic double bonds (Scheme 3).

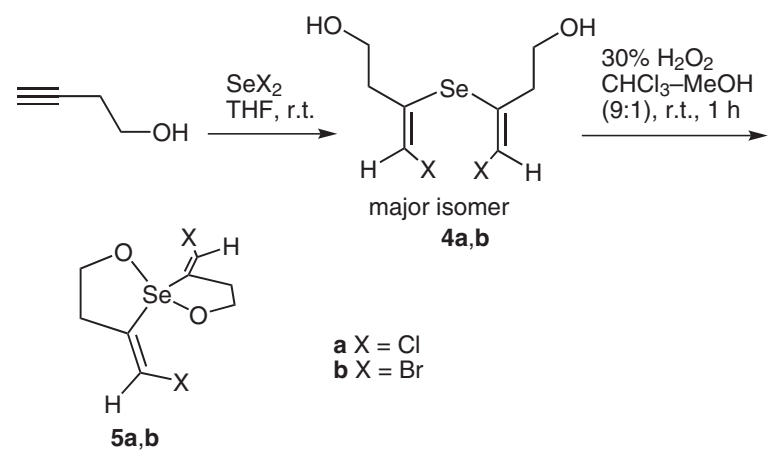

Scheme 3 Reaction of homopropargyl alcohol with $\mathrm{SeCl}_{2}$ and $\mathrm{SeBr}_{2}$

In an alternative approach to the preparation of spirodioxaselenuranes, we have found that but-2-yn-1,4-diol smoothly reacts with selenium dihalides in a completely stereospecific manner and affords the corresponding water-soluble tetrahydroxymethyldivinyl selenides $\mathbf{6 a}, \mathbf{b}$ in high yields. Noteworthy is the fact that unlike the syn-addition of $\mathrm{SeX}_{2}$ to the triple bond of propargyl alcohols containing a single hydroxymethyl functionality, but-2yn-1,4-diol gives under the similar conditions exclusively 1,2-anti-adducts. The mechanistic explanation of this striking difference is still under investigation. However, in general anti-addition of electrophilic selenium reagents to multiple bonds is the expected stereochemical result. ${ }^{17}$ Compounds 6 upon oxidation with 30\% hydrogen peroxide in aqueous solution produce the spiroselenurane compounds 7 with the endocyclic double bonds in excellent yields (Scheme 4). Spiroselenurane compounds precipi- tated from the reaction mixture as white needles within 15 minutes, and were recrystallized from water and fully characterized by spectroscopic methods. The structure of the chloro-substituted compound 7a was confirmed by Xray crystallography (Figure 2).

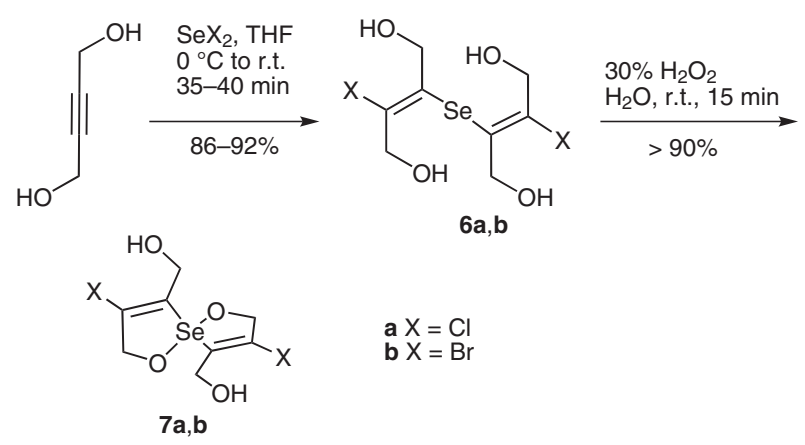

Scheme 4 Formation of dioxydroxymethyl spiroselenuranes

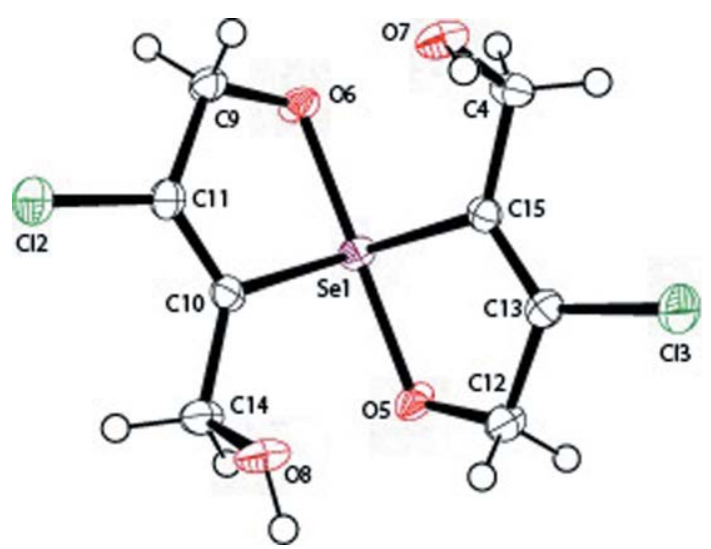

Figure 2 X-ray structure of (3,8-dichloro-9-hydroxymethyl-1,6-dioxa-5-selenaspiro[4.4]nona-3,8 diene-4-yl)methanol (7a); ORTEP diagram of spiroselenurane $7 \mathbf{a}^{18}$

The reaction of substituted alkyne diols with selenium dihalogenides was used for the preparation of a series of spirodioxaselenuranes. However, in the case of 2,5dimethylhex-3-yne-2,5-diol the reaction with $\mathrm{SeCl}_{2}$ proceeded slowly and produced, not a divinyl selenide, but the seleninate ester $\mathbf{9}$, presumably via 8 (Scheme 5). Steric hindrance of the four methyl groups precluded addition to the triple bond. ${ }^{16}$
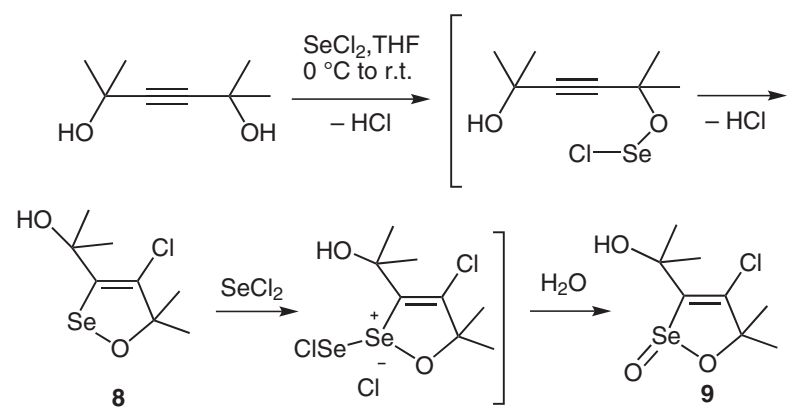

Scheme 5 Formation of cyclic seleninate ester 9 
Recently, we have shown that the addition of commercially available selenium tetrachloride to the triple bond of propargyl alcohols proceeded easily with the same regioand stereochemistry as selenium dichloride and produces unstable divinylselenium dichloride intermediates. ${ }^{19}$ The latter underwent hydrolysis to the corresponding divinyl selenoxides during basic workup. ${ }^{19}$ In the case in hand, but-2-yn-1,4-diol reacted stereospecifically with selenium tetrachloride in anhydrous acetonitrile and afforded the expected spiroselenurane compound 7a in a one-pot manner and in good yield (Scheme 6).

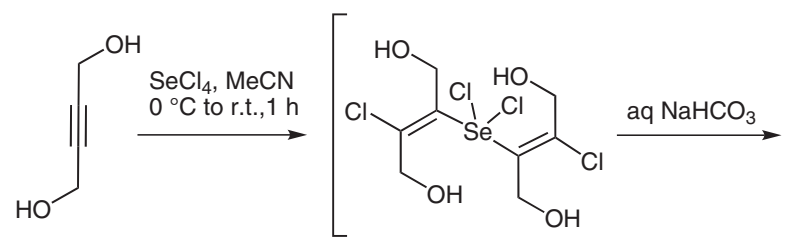<smiles>OCC(O)=C(CO)C(CO)=C(CO)CO</smiles>

Scheme 6 One-pot preparation of dihydroxymethyl spiroselenuranes

Even the sterically hindered 2,5-dimethylhex-3-yne-2,5diol reacted easily with $\mathrm{SeCl}_{4}$ and produced, in an almost 1:1 ratio, two products, $\mathbf{1 0}$ and $\mathbf{1 1}$, which were separated by column chromatography (Scheme 7). The first product, selenium-bridged dihydrofuran derivative 10, presumably was formed via $s y n$-addition of $\mathrm{SeCl}_{4}$ to the triple bond followed by double intramolecular dehydrative cyclization. The second one 11, was identified as a chlorinated analogue of the seleninate ester 9. Dicylic selenide $\mathbf{1 0}$ upon oxidation with hydrogen peroxide gave the expected stable selenoxide 12 (Scheme 8). The structure of the latter was unambiguously confirmed by X-ray crystallography (Figure 3).

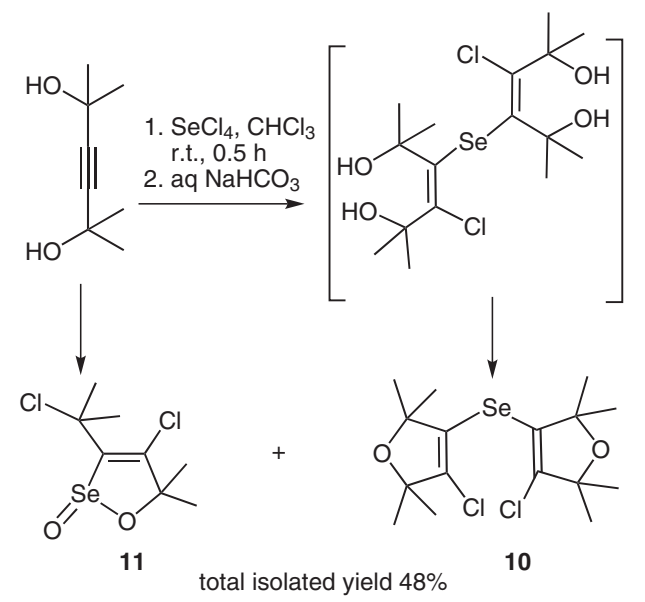

Scheme 7 Reaction of 2,5-dimethylhex-3-yne-2,5-diol with $\mathrm{SeCl}_{4}$

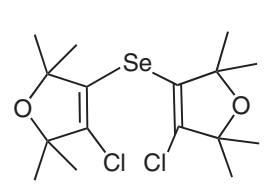

10

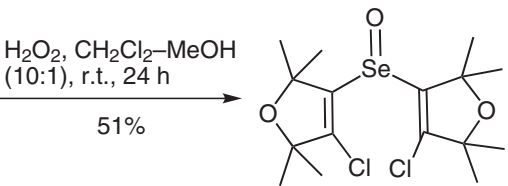

12
Scheme 8 Oxidation of dihydrofuran selenide derivative $\mathbf{1 0}$

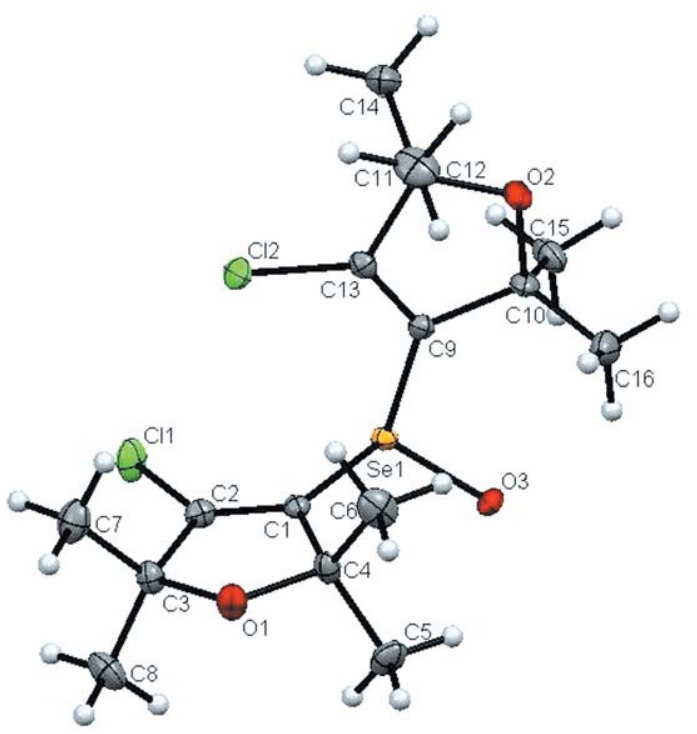

Figure 3 X-ray structure of [bis(4-chloro-2,2,5,5-tetramethyl-2,5dihydrofuran-3-yl]seleninyl 12; ORTEP diagram of selenoxide $\mathbf{1 2}^{18}$

An interesting result was obtained using ethyl 4-hydroxybut-2-ynoate (13) as a hydroxyalkyne substrate. Whereas selenium dichloride does not react with this alcohol, selenium tetrachloride undergoes smooth 1,2-addition to the deactivated triple bond of this propargyl alcohol. However, the regiochemistry differ from the one observed in a 'normal' $\mathrm{SeCl}_{2}$ addition (see, Scheme 1) and produces, after hydrolysis of the intermediate, the divinyl selenium oxide 14 with the geometry that permits its subsequent cyclization to the spiro derivative 15 (Scheme 9).

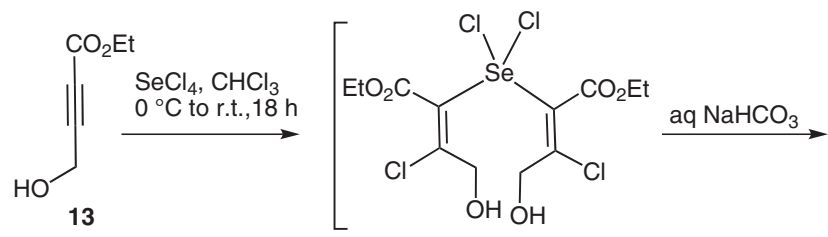<smiles>CCOC(=O)C(=C(Cl)CO)[Se](=O)C(CO)=C(Cl)CO</smiles>

14<smiles>CCOC1=C(Cl)CO[Se]1(OCC)(OCC)C(C)=O</smiles>

15

Scheme 9 Reaction of ethyl 4-hydroxybut-2-ynoate (13) with $\mathrm{SeCl}_{4}$ 
The novel selenium-containing spiro compounds $\mathbf{5 a}, \mathbf{b}$ and $\mathbf{7 a}, \mathbf{b}$ were found to exhibit higher glutathione peroxidase mimetic activity than the widely studied compound ebselen (in nonenzymic conditions). To examine the glutathione peroxidase-like (GPx) catalytic activity of the spiroselenurane compounds, $30 \% \mathrm{H}_{2} \mathrm{O}_{2}$ and benzylthiol $(\mathrm{BnSH})$ were chosen as the oxidant and stoichiometric reductant, respectively. The oxidation of $\mathrm{BnSH}$ to the disulfide $\mathrm{BnSSBn}$ was monitored by ${ }^{1} \mathrm{H}$ NMR spectroscopy. When $10 \mathrm{~mol} \%$ of the catalyst $6 \mathbf{a}$ or $7 \mathbf{a}$ was used in the presence of excess $30 \% \mathrm{H}_{2} \mathrm{O}_{2}, 75 \%$ of $\mathrm{BnSH}$ was converted into BnSSBn after 1.5 hours. (3Z)-4-Chloro-3- $\{[(Z)-2-$ chloro-1-(1-hydroxy-1-methylethyl)vinyl]selanyl $\}-2-$ methylbut-3-en-2-ol (1a) exhibits a similar GPx-like catalytic activity. In the control experiment in the absence of the catalyst under the same reaction condition after 24 hours, only $4 \%$ of BnSSBn was observed in the reaction mixture according to ${ }^{1} \mathrm{H}$ NMR spectrum. The suggested catalytic cycle of compounds $\mathbf{6}$ and $\mathbf{7}$ is shown on Scheme 10.

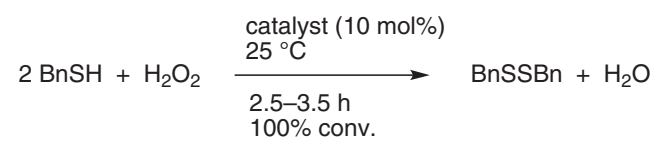

catalyst $=\mathbf{1 a} ; \mathbf{5 a}, \mathbf{b} ; \mathbf{6 a}, \mathbf{b} ; \mathbf{7 a}, \mathbf{b}$

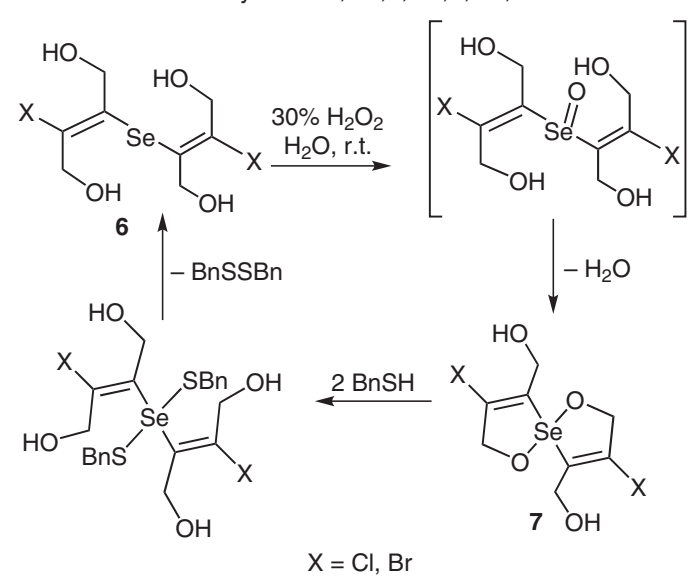

Scheme 10 GPx-like activity of divinyl selenium systems

Thus, an easy and efficient synthesis of the water-soluble divinyl selenides has been achieved. These divinyl selenides perform as glutathione peroxidase mimetics with high efficacy. Spiroselenurane 7a also showed some antifungal activity in preliminary experiments.

The THF solution of $\mathrm{SeCl}_{2}$ was prepared by the known procedure ${ }^{14}$ and used immediately. All solvents and reagents were obtained from Aldrich or Fluka and used without further purification with the following exception: THF was distilled from sodium benzophenone dianion just before use and $\mathrm{CHCl}_{3}$ was distilled from $\mathrm{P}_{2} \mathrm{O}_{5}$. All reactions were carried out under dry argon atmosphere using ovendried glassware. Reagents and solvents were handled by using standard syringe-septum cap techniques. Column chromatography was performed with Merck silica gel 60 (230-400 mesh), and TLC was run on precoated Merck silica gel plates $60 \mathrm{~F}_{254}(2.00 \mathrm{~mm})$. Preparative TLC was carried out in glass sheets precoated with Merck sil- ica gel $60 \mathrm{~F}_{254}(0.25 \mathrm{~mm})$. All new compounds have satisfactory analytical and spectroscopic data.

Melting points were obtained on a Thomas Hoover melting point apparatus and are uncorrected. IR spectra were recorded on a Bruker Tensor 27 FTIR instrument. ${ }^{1} \mathrm{H}$ NMR and ${ }^{13} \mathrm{C}$ NMR were recorded on Bruker DPX-300, DMX-600 or Avance-III-700 spectrometers in either $\mathrm{CDCl}_{3}$ or other deuterated solvents, using TMS as internal standard. Chemical shifts are reported in $\delta$ units, and coupling constants in Hz. COSY and NOSY experiments have been carried out in order to assign ${ }^{1} \mathrm{H}$ and ${ }^{13} \mathrm{C}$ spectra and confirmed the structures of new compounds. Mass spectra were obtained on Auto flex Tof/Tof Bruker MALDI (matrix assisted laser desorption ionization) instrument with graphite matrix. High-resolution mass spectra were obtained on a VG-Fison Autospec instrument. Elemental analyses were performed on Thermo CHNS Analyzer FlashEA instrument.

Divinyl selenides 1a-d were prepared according to our reported procedure. ${ }^{15,16}$ Ethyl 4-hydroxybut-2-ynoate (13) was prepared by the known procedure. ${ }^{20}$

Oxidation of Divinyl Selenides with TBHP; General Procedure An excess of TBHP $(0.90 \mathrm{~mL}, 5 \mathrm{mmol}, \sim 5.5 \mathrm{M}$ solution in nonane $)$ was added to divinyl selenide $\mathbf{1 a}-\mathbf{d}(1 \mathrm{mmol})$ dissolved in $\mathrm{CH}_{2} \mathrm{Cl}_{2}$ $(10 \mathrm{~mL})$ and the solution was stirred at r.t. for $18 \mathrm{~h}$. After completion of the reaction (TLC, eluent: hexanes-EtOAc, 4:1), the solvent was evaporated to dryness under reduced pressure. All selenoxides 2a-d were purified by removing the excess of TBHP overnight under vacuum. In the case of stable compounds, further purification can be done by column chromatography, if required, using EtOAc$\mathrm{MeOH}(4: 1)$ as eluent.

(3Z)-4-Chloro-3-\{[(Z)-2-chloro-1(1-hydroxy-1-methylethyl)vinyl]seleninyl\}-2-methylbut-3-en-2-ol (2a) Yield: $0.294 \mathrm{~g} \mathrm{(88 \% )}$; white solid; $\mathrm{mp} 126^{\circ} \mathrm{C}$.

IR (KBr): 3355, 2936, 2560, 1604, 1466, 1426, 1370, 1305, 1173, $1149,981,901,821,786 \mathrm{~cm}^{-1}$.

${ }^{1} \mathrm{H} \mathrm{NMR}\left(300 \mathrm{MHz}, \mathrm{CDCl}_{3}\right): \delta=1.51(\mathrm{~s}, 6 \mathrm{H}), 1.73(\mathrm{~s}, 6 \mathrm{H}), 5.67$ (br s, $2 \mathrm{H}), 6.73$ (s, $2 \mathrm{H})$.

${ }^{13} \mathrm{C}$ NMR $\left(75 \mathrm{MHz}, \mathrm{CDCl}_{3}\right): \delta=27.8\left(\mathrm{CH}_{3}\right), 28.4\left(\mathrm{CH}_{3}\right), 73.6$ $\left[{ }^{2} J_{\mathrm{C}, \mathrm{Se}}=12.2 \mathrm{~Hz},\left(\mathrm{CH}_{3}\right)_{2} \mathrm{C}\right], 122.4(=\mathrm{CCl}), 157.1\left({ }^{1} J_{\mathrm{C}, \mathrm{Se}}=123.6 \mathrm{~Hz}\right.$, $=\mathrm{CSe}$ ).

HRMS: $m / z[\mathrm{M}+\mathrm{H}]$ calcd for $\mathrm{C}_{10} \mathrm{H}_{17} \mathrm{Cl}_{2} \mathrm{O}_{3}{ }^{80} \mathrm{Se}: 334.9720$; found: 334.9720 .

Anal. Calcd for $\mathrm{C}_{10} \mathrm{H}_{16} \mathrm{Cl}_{2} \mathrm{O}_{3} \mathrm{Se}: \mathrm{C}, 35.95 ; \mathrm{H}, 4.83 ; \mathrm{O}, 14.37$. Found: C, 35.56; H, 4.76; O, 14.35.

(3Z)-4-Bromo-3-\{[(Z)-2-bromo-1(1-hydroxy-1-methylethyl)vinyl]seleninyl\}-2-methylbut-3-en-2-ol (2b)

Yield: $0.368 \mathrm{~g}(87 \%)$; white solid; $\mathrm{mp} 112^{\circ} \mathrm{C}$.

${ }^{1} \mathrm{H}$ NMR (300 MHz, $\left.\mathrm{CDCl}_{3}\right): \delta=1.54$ (s, $\left.6 \mathrm{H}\right), 1.75$ (s, $\left.6 \mathrm{H}\right), 6.31$ (br s, $2 \mathrm{H}), 6.74$ (s, $2 \mathrm{H})$.

${ }^{13} \mathrm{C} \mathrm{NMR}\left(75 \mathrm{MHz}, \mathrm{CDCl}_{3}\right): \delta=27.7\left(\mathrm{CH}_{3}\right), 28.4\left(\mathrm{CH}_{3}\right), 74.3(\mathrm{C})$, $110.3(\mathrm{C}=), 158.8(\mathrm{C}=)$.

(2Z)-3-Chloro-2-\{[(1Z)-2-chloro-1-(hydroxymethyl)prop-1enyl]seleninyl $\}$ but-2-en-1-ol (2c)

Eluent EtOAc-MeOH (4:1); yield: 0.260 g (85\%); white solid; mp $81-83{ }^{\circ} \mathrm{C}$.

IR (KBr): 3102, 1623, 1434, 1373, 1131, 1007, $806 \mathrm{~cm}^{-1}$.

${ }^{1} \mathrm{H} \mathrm{NMR}\left(300 \mathrm{MHz}, \mathrm{CDCl}_{3}\right): \delta=2.42(\mathrm{~s}, 6 \mathrm{H}), 4.68(\mathrm{br} \mathrm{s}, 2 \mathrm{H})$, ABq: $4.58(\mathrm{~d}, J=15.4 \mathrm{~Hz}, 2 \mathrm{H}), 4.70(\mathrm{~d}, J=15.4 \mathrm{~Hz}, 2 \mathrm{H})$.

${ }^{13} \mathrm{C}$ NMR $\left(75 \mathrm{MHz}, \mathrm{CDCl}_{3}\right): \delta=25.03\left(\mathrm{CH}_{3}\right), 58.06\left(\mathrm{CH}_{2}\right), 137.02$ $(\mathrm{C}=), 140.43(\mathrm{C}=)$.

(2Z)-3-Chloro-2-\{[(Z)-2-chloro-1-(hydroxymethyl)vinyl]seleninyl\}prop-2-en-1-ol) (2d)

Yield: $0.228 \mathrm{~g}(82 \%)$; viscous liquid. 
${ }^{1} \mathrm{H}$ NMR $\left(300 \mathrm{MHz}\right.$, acetone- $\left.d_{6}\right): \delta=4.22(\mathrm{~s}, 2 \mathrm{H}), \mathrm{ABq}: 4.53$ (dd, $J=14.3,1.5 \mathrm{~Hz}, 2 \mathrm{H}), 4.73(\mathrm{dd}, J=14.3,1.5 \mathrm{~Hz}, 2 \mathrm{H}), 7.02(\mathrm{t}, 1.5$ $\mathrm{Hz}, 2 \mathrm{H})$.

${ }^{13} \mathrm{C}$ NMR (75 MHz, acetone- $\left.d_{6}\right): \delta=57.4\left(\mathrm{CH}_{2}\right), 123.6(\mathrm{CH}=)$, $146.4\left({ }^{1} J_{\mathrm{CSe}}=136.2 \mathrm{~Hz}, \mathrm{C}=\right)$.

(\{I(2Z,2'Z)-Seleninylbis(3-chloroprop-2-ene-2,1-diyl)|bis(oxy) $\}$ bis(methylene)) dibenzene (2e)

Eluent: EtOAc-hexane (1:1); yield: $0.504 \mathrm{~g}(93 \%)$; viscous liquid.

${ }^{1} \mathrm{H}$ NMR (300 MHz, $\mathrm{CDCl}_{3}$ ): $\delta=$ superposition of two ABq: $\mathrm{ABq}$ $4.28(\mathrm{~d}, J=13.1 \mathrm{~Hz}, 2 \mathrm{H}), 4.45(\mathrm{~d}, J=13.1 \mathrm{~Hz}, 2 \mathrm{H})$ and $\mathrm{ABq} 4.40$ $(\mathrm{d}, J=11.6 \mathrm{~Hz}, 2 \mathrm{H}), 4.47(\mathrm{~d}, J=11.6 \mathrm{~Hz}, 2 \mathrm{H}), 6.85(\mathrm{~s}, 2 \mathrm{H}), 7.29$ $(\mathrm{m}, 10 \mathrm{H})$.

${ }^{13} \mathrm{C} \mathrm{NMR}\left(75 \mathrm{MHz}, \mathrm{CDCl}_{3}\right): \delta=63.80\left({ }^{2} J_{\mathrm{C}, \mathrm{Se}}=6.8 \mathrm{~Hz}, \mathrm{CH}_{2} \mathrm{CSe}\right)$ $72.98\left(\mathrm{CH}_{2} \mathrm{Ph}\right), 125.81\left({ }^{2} J_{\mathrm{C}, \mathrm{Se}}=21 \mathrm{~Hz}, \mathrm{CH}=\right), 127.67(\mathrm{CH}), 128.28$ (CH), 136.67 (C-ipso), $142.17\left({ }^{1} J_{\mathrm{C}, \mathrm{Se}}=125.4 \mathrm{~Hz}, \mathrm{C}=\right)$.

HRMS (DCI $\left.+\mathrm{CH}_{4}\right): m / z[\mathrm{M}+\mathrm{H}]^{+}$calcd for $\mathrm{C}_{20} \mathrm{H}_{21} \mathrm{Cl}_{2} \mathrm{O}_{3}{ }^{80} \mathrm{Se}$ : 459.0033; found: 459.0071 .

\section{Oxidation of Divinyl Selenide 1a with MCPBA; Preparation of} Compounds $2 \mathrm{a}$ and 3

4-Chloro-3-[2-chloro-1-(1-hydroxy-1-methylethyl)vinylselanyl]-

2-methylbut-3-en-2-ol (1a; $318 \mathrm{mg}, 1 \mathrm{mmol})$ was dissolved in $\mathrm{CH}_{2} \mathrm{Cl}_{2}(10 \mathrm{~mL})$ and a $\mathrm{CH}_{2} \mathrm{Cl}_{2}$ solution $(15 \mathrm{~mL})$ of MCPBA $(77 \%$, $491 \mathrm{mg}, 2.2 \mathrm{mmol}$ ) was added slowly. The reaction mixture was then stirred for $24 \mathrm{~h}$ at r.t. TLC (eluent: EtOAc- $\mathrm{MeOH}, 4: 1$ ) indicated the formation of two products. The mixture was then quenched with sat. aq $\mathrm{Na}_{2} \mathrm{SO}_{3}(10 \mathrm{~mL})$ under ice cold conditions. The product was extracted with $\mathrm{CH}_{2} \mathrm{Cl}_{2}(30 \mathrm{~mL})$, and the $\mathrm{CH}_{2} \mathrm{Cl}_{2}$ layer was washed with sat. aq $\mathrm{NaHCO}_{3}(5 \mathrm{~mL}), \mathrm{H}_{2} \mathrm{O}(10 \mathrm{~mL})$, and brine $(10 \mathrm{~mL})$. Drying $\left(\mathrm{MgSO}_{4}\right)$ and evaporation of the solvent under reduced pressure produced the crude mixture of corresponding selenoxide $\mathbf{2 a}$ and selenone $\mathbf{3}$. The products were separated by column chromatography using EtOAc- $\mathrm{MeOH}$ (80:20) as an eluent. The selenone, which is less polar than selenoxide eluted first, followed by selenoxide. Overall isolated yield $(0.333 \mathrm{~g}, 97 \%)$; product distribution selenone/selenoxide $=65: 35$.

For the physical and spectral data of $\mathbf{2 a}$, see above.

(3Z)-4-Chloro-3-\{[(Z)-2-chloro-1(1-hydroxy-1-methylethyl)vinyl]selenonyl\}-2-methylbut-3-en-2-ol (3)

Yield: $0.22 \mathrm{~g}(63 \%)$; white solid; $\mathrm{mp} 160-162{ }^{\circ} \mathrm{C}$.

IR (KBr): 3342, 3052, 2495, 1603, 1459, 1369, 1309, 1170, 1134, $979,901,867,838 \mathrm{~cm}^{-1}$.

${ }^{1} \mathrm{H} \mathrm{NMR}\left(300 \mathrm{MHz}, \mathrm{CDCl}_{3}\right): \delta=1.72(\mathrm{~s}, 12 \mathrm{H}), 3.42$ (br s, $\left.2 \mathrm{H}\right)$, 7.44 (s, $2 \mathrm{H})$.

${ }^{13} \mathrm{C} \mathrm{NMR}\left(75 \mathrm{MHz}, \mathrm{CDCl}_{3}\right): \delta=28.8\left(\mathrm{CH}_{3}\right), 74.1(\mathrm{C}), 130.3(\mathrm{C}=)$, $157.3(\mathrm{C}=)$.

HRMS: $m / z[\mathrm{M}+\mathrm{H}]$ calcd for $\mathrm{C}_{10} \mathrm{H}_{17} \mathrm{Cl}_{2} \mathrm{O}_{4}{ }^{78} \mathrm{Se}: 350.9669$; found: 350.9647 .

Anal. Calcd for $\mathrm{C}_{10} \mathrm{H}_{16} \mathrm{Cl}_{2} \mathrm{O}_{4} \mathrm{Se}: \mathrm{C}, 34.31 ; \mathrm{H}, 4.61$. Found: $\mathrm{C}, 34.42$; H, 4.63.

\section{Oxidation of Divinyl Selenides with $\mathrm{H}_{2} \mathrm{O}_{2}$; Preparation of 5a,} $5 b$, and 12

The corresponding divinyl selenide $4 \mathbf{a}, \mathbf{4 b}$, or 10 (0.545 $\mathrm{mmol})$ was dissolved in a mixture of $\mathrm{CHCl}_{3}$ and $\mathrm{MeOH}(9: 1,10 \mathrm{~mL})$, respectively, and $30 \%$ aq $\mathrm{H}_{2} \mathrm{O}_{2}(0.05 \mathrm{~mL}, 1.635 \mathrm{mmol})$ was added. The mixture was stirred for $1 \mathrm{~h}$ at r.t., the solvent was evaporated, and the product was isolated by silica gel column chromatography.

\section{(4Z,9Z)-4,9-Bis(chloromethylene)-1,6-dioxa-5 $\lambda^{4}$-selenospi-}

ro[4,4]nonane (5a)

Eluent: EtOAc-MeOH (8:1); yield: 0.06 g (40\%); white solid; mp $78-80{ }^{\circ} \mathrm{C}$.

IR (neat): 3396 (br), 2955, 2116, 1614, 1420, 1260, 1062, 778, 417 $\mathrm{cm}^{-1}$.
${ }^{1} \mathrm{H}$ NMR $\left(700 \mathrm{MHz}, \mathrm{CDCl}_{3}\right): \delta=2.79(\mathrm{~m}, 4 \mathrm{H}), 4.02(\mathrm{dt}, J=9.6,6.1$ $\mathrm{Hz}, 2 \mathrm{H}), 4.17(\mathrm{dt}, J=9.6,6.1 \mathrm{~Hz}, 2 \mathrm{H}), 7.17(\mathrm{t}, J=2.5 \mathrm{~Hz}, 2 \mathrm{H})$.

${ }^{13} \mathrm{C}$ NMR $\left(175 \mathrm{MHz}, \mathrm{CDCl}_{3}\right): \delta=31.6\left(\mathrm{CH}_{2}\right), 64.3\left(\mathrm{CH}_{2}\right), 125.6$ $\left({ }^{2} J_{\mathrm{C}, \mathrm{Se}}=25 \mathrm{~Hz}, \mathrm{C}_{\mathrm{q}}-\mathrm{Cl}\right), 138.5\left({ }^{1} J_{\mathrm{C}, \mathrm{Se}}=101.5 \mathrm{~Hz}, \mathrm{C}_{\mathrm{q}}-\mathrm{Se}\right)$.

MS (DCI/CH $): m / z(\%)=288.9$ (78), 252.9 (26), 236.9 (14), 206.9 (17), 183.9 (79), 168.9 (14), 123.0 (15), 83.9 (100).

HRMS: $m / z\left[\mathrm{M}^{+}\right]$calcd for $\mathrm{C}_{8} \mathrm{H}_{10} \mathrm{Cl}_{2} \mathrm{O}_{2} \mathrm{Se}$ : 287.9223; found: 288.0302 .

\section{(4Z,9Z)-4,9-Bis(bromomethylene)-1,6-dioxa-5 $\lambda^{4}$-selenospi-}

ro[4,4]nonane $(5 b)$

Eluent: EtOAc-MeOH (8:1); yield: 0.18 g (89\%); white solid; mp $65-67^{\circ} \mathrm{C}$.

${ }^{1} \mathrm{H}$ NMR $\left(700 \mathrm{MHz}, \mathrm{CDCl}_{3}\right): \delta=2.77(\mathrm{~m}, 4 \mathrm{H}), 4.02(\mathrm{ddd}, J=9.6$, 7.5, $6.0 \mathrm{~Hz}, 2 \mathrm{H}) 4.18$ (dddd, $J=9.6,7.5,6.0,0.14 \mathrm{~Hz}, 2 \mathrm{H}), 7.40$ $(\mathrm{t}, J=2.5 \mathrm{~Hz}, 2 \mathrm{H})$.

${ }^{13} \mathrm{C} \mathrm{NMR}\left(175 \mathrm{MHz}, \mathrm{CDCl}_{3}\right): \delta=33.6\left(\mathrm{CH}_{2}\right), 64.2\left(\mathrm{CH}_{2} \mathrm{O}\right), 114.1$ $\left({ }^{2} J_{\mathrm{C}, \mathrm{Se}}=22.6 \mathrm{~Hz}, \mathrm{CH}\right), 140.0\left({ }^{1} J_{\mathrm{C}, \mathrm{Se}}=103.0 \mathrm{~Hz}, \mathrm{C}_{\mathrm{q}}\right)$.

MS $\left(\mathrm{CI} / \mathrm{CH}_{4}\right): m / z(\%)=378.8(88), 296.9$ (13), 227.8 (100), 118.9 (8).

HRMS: $m / z\left[\mathrm{M}^{+}\right]$calcd for $\mathrm{C}_{8} \mathrm{H}_{10} \mathrm{Br}_{2} \mathrm{O}_{2} \mathrm{Se}: 375.8213$; found: 375.8251 .

Bis(4-chloro-2,2,5,5-tetramethyl-2,5-dihydrofuran-3-yl)selenenyl (12)

Eluent: EtOAc-hexane (1:3); yield: $0.115 \mathrm{~g}$ (51\%); colorless plates; mp 96-97 ${ }^{\circ} \mathrm{C}\left(\mathrm{CHCl}_{3}\right.$-hexane).

${ }^{1} \mathrm{H}$ NMR $\left(\mathrm{CDCl}_{3}, 600 \mathrm{MHz}\right): \delta=1.40(\mathrm{~s}, 6 \mathrm{H}), 1.44$ (s, $\left.6 \mathrm{H}\right), 1.62$ (s, $12 \mathrm{H})$.

${ }^{13} \mathrm{C} \mathrm{NMR}\left(\mathrm{CDCl}_{3}, 150 \mathrm{MHz}\right): \delta=27.3\left(\mathrm{CH}_{3}\right), 27.4\left(\mathrm{CH}_{3}\right), 29.0$ $\left(\mathrm{CH}_{3}\right), 30.4\left(\mathrm{CH}_{3}\right), 86.1\left(\mathrm{Me}_{2} \mathrm{C}\right), 88.9\left(\mathrm{Me}_{2} \mathrm{C}\right), 134.3\left({ }^{1} J_{\mathrm{C}, \mathrm{Se}}=150.6\right.$ $\mathrm{Hz},=\mathrm{CSe}), 143.02\left({ }^{2} J_{\mathrm{C}, \mathrm{Se}}=16.5 \mathrm{~Hz},=\mathrm{CCl}\right)$.

MS (DCI): $m / z(\%)=415.0\left(100,[\mathrm{M}+\mathrm{H}]^{+}\right), 399.0(26), 381$ (9.37), 159.1 (13.3), 85.0 (48.7).

HRMS: $m / z[\mathrm{M}+\mathrm{H}]^{+}$calcd for $\mathrm{C}_{16} \mathrm{H}_{25} \mathrm{O}_{3}{ }^{35} \mathrm{Cl}^{37} \mathrm{Cl}^{78} \mathrm{Se}$ : 415.0324; found: 415.0317.

Anal. Calcd for $\mathrm{C}_{16} \mathrm{H}_{24} \mathrm{O}_{3} \mathrm{Cl}_{2} \mathrm{Se}$ : C, 46.39; $\mathrm{H}, 5.84$. Found: C, 46.67; H, 5.89.

\section{Divinyltetrahydroxy Selenides 6a,b}

The THF solution of $\mathrm{SeCl}_{2}(1 \mathrm{mmol})$ prepared by the known procedure $^{14}$ was added dropwise to a solution of but-2-yn-1,4-diol $(172 \mathrm{mg}, 2 \mathrm{mmol})$ in anhydrous THF $(2 \mathrm{~mL})$ at $0{ }^{\circ} \mathrm{C}$. The reaction mixture was then stirred at r.t. for $45 \mathrm{~min}$. After completion of the reaction (TLC, eluent: EtOAc), the mixture was extracted with EtOAc $(20 \mathrm{~mL})$ and the EtOAc layer was washed with brine $(2 \times 5$ $\mathrm{mL})$ and dried $\left(\mathrm{MgSO}_{4}\right)$. After evaporation of the solvent, a crude product containing some black gummy materials and selenide compounds was obtained. This crude product was dissolved in a minimum amount of $\mathrm{H}_{2} \mathrm{O}$ and filtered. The filtrate contained the pure selenide $\mathbf{6 a}$, which was isolated as a light yellowish solid after lyophilization. The corresponding bromo derivative $\mathbf{6 b}$ was prepared by the same procedure using $\mathrm{SeBr}_{2}{ }^{21}$ instead of $\mathrm{SeCl}_{2}$.

2-Chloro-3-(2-chloro-3-hydroxy-1-hydroxymethylpropenylselanyl)but-2-ene-1-4-diol (6a)

Yield: $0.296 \mathrm{~g} \mathrm{(92 \% );} \mathrm{yellowish} \mathrm{solid;} \mathrm{mp} 87-89^{\circ} \mathrm{C}$.

IR (KBr): 3300 (br), 2923, 2873, 1597, 1481, 1439, 1356, 1124, $1073,1004,934 \mathrm{~cm}^{-1}$.

${ }^{1} \mathrm{H}$ NMR (300 MHz, $\left.\mathrm{D}_{2} \mathrm{O}\right): \delta=4.42(\mathrm{~s}, 4 \mathrm{H}), 4.63(\mathrm{~s}, 4 \mathrm{H})$.

${ }^{13} \mathrm{C} \mathrm{NMR}\left(75 \mathrm{MHz}, \mathrm{D}_{2} \mathrm{O}\right): \delta=62.6\left(\mathrm{CH}_{2}\right), 64.8\left(\mathrm{CH}_{2}\right), 128.8(\mathrm{C}=)$, $137.5(\mathrm{C}=)$.

HRMS: $m / z[\mathrm{M}+\mathrm{H}]$ calcd for $\mathrm{C}_{8} \mathrm{H}_{13} \mathrm{Cl}_{2} \mathrm{O}_{4}{ }^{80} \mathrm{Se}: 322.9356$; found: 322.9306 . 
Anal. Calcd for $\mathrm{C}_{8} \mathrm{H}_{12} \mathrm{Cl}_{2} \mathrm{O}_{4} \mathrm{Se}$ : C, 29.84: $\mathrm{H}, 3.76$; O, 19.87. Found: C, 30.08; H, 3.60; O, 18.6.

2-Bromo-3-(2-bromo-3-hydroxy-1-hydroxymethylpropenylselanyl)but-2-ene-1-4-diol (6b)

Yield: $0.37 \mathrm{~g}(90 \%)$; colorless solid; $\mathrm{mp} 103-105^{\circ} \mathrm{C}$.

IR (KBr): 3301 (br), 2923, 2873, 1597, 1481, 1439, 1356, 1124, $1073,1004,934 \mathrm{~cm}^{-1}$.

${ }^{1} \mathrm{H}$ NMR (300 MHz, DMSO- $\left.d_{6}\right): \delta=4.22$ (s, $\left.4 \mathrm{H}\right), 4.40$ (s, $\left.4 \mathrm{H}\right)$.

${ }^{13} \mathrm{C}$ NMR $\left(75 \mathrm{MHz}, \mathrm{DMSO}-d_{6}\right): \delta=66.1\left(\mathrm{CH}_{2}\right), 67.2\left(\mathrm{CH}_{2}\right), 128.9$ $(\mathrm{C}=), 132.6(\mathrm{C}=)$.

HRMS: $m / z[\mathrm{M}+\mathrm{H}]$ calcd for $\mathrm{C}_{8} \mathrm{H}_{13} \mathrm{Br}_{2} \mathrm{O}_{4}{ }^{80} \mathrm{Se}: 322.9356$; found: 322.9306 .

Anal. Calcd for $\mathrm{C}_{8} \mathrm{H}_{12} \mathrm{Br}_{2} \mathrm{O}_{4}$ Se: C, 29.84; H, 3.76. Found: C, 30.08; $\mathrm{H}, 3.60$.

\section{Spiroselenuranes $7 \mathbf{a}, \mathbf{b}$}

The corresponding divinyl selenide $\mathbf{6 a}$ or $\mathbf{6 b}(0.545 \mathrm{mmol})$ was dissolved in $\mathrm{H}_{2} \mathrm{O}(10 \mathrm{~mL})$ and $30 \%$ aq $\mathrm{H}_{2} \mathrm{O}_{2}(0.05 \mathrm{~mL}, 1.635 \mathrm{mmol})$ was added. The mixture was stirred for $15 \mathrm{~min}$ at r.t. whereupon a colorless solid precipitated. The solid was collected by filtration, washed with distilled $\mathrm{H}_{2} \mathrm{O}(5 \mathrm{~mL})$, and dried under vacuum overnight to obtain the pure product $7 \mathbf{a}$ or $7 \mathbf{b}$, respectively.

\section{(3,8-Dichloro-9-hydroxymethyl-1,6-dioxa-5 $\lambda^{4}$-selenaspi-} ro[4.4] nona-3,8-dien-4-yl)methanol (7a)

Yield: $0.16 \mathrm{~g}(93 \%)$; colorless needles; $\mathrm{mp} 151{ }^{\circ} \mathrm{C}\left(\mathrm{H}_{2} \mathrm{O}\right)$.

IR (KBr): 3392 (br), 2914, 1641, 1422, 1284, 1227, 1094, 1022, 952 $\mathrm{cm}^{-1}$.

${ }^{1} \mathrm{H}$ NMR $\left(300 \mathrm{MHz}, \mathrm{DMSO}-d_{6}\right): \delta=4.28(\mathrm{dd}, J=12.6,4.2 \mathrm{~Hz}, 2$ H), $4.44(\mathrm{dd}, J=12.6,6.3 \mathrm{~Hz}, 2 \mathrm{H}), 4.66(\mathrm{~d}, J=16.2 \mathrm{~Hz}, 2 \mathrm{H}), 5.02$ $(\mathrm{d}, J=16.2 \mathrm{~Hz}, 2 \mathrm{H}), 5.12(\mathrm{t}, J=6.0 \mathrm{~Hz}, 2 \mathrm{H})$.

${ }^{13} \mathrm{C}$ NMR (75MHz, DMSO- $d_{6}$ ): $\delta=56.6(2 \mathrm{C}), 75.7$ (2 C), 134.7 (2 C), 142.8 (2 C).

HRMS: $m / z[\mathrm{M}+\mathrm{H}]^{+}$calcd for $\mathrm{C}_{8} \mathrm{H}_{11} \mathrm{Cl}_{2} \mathrm{O}_{4}{ }^{80} \mathrm{Se}: 320.9199$; found: 320.9200 .

Anal. Calcd for $\mathrm{C}_{8} \mathrm{H}_{10} \mathrm{Cl}_{2} \mathrm{O}_{4}$ Se: $\mathrm{C}, 30.02 ; \mathrm{H}, 3.15 ; \mathrm{O}, 20.00$. Found: C, 30.32; H, 3.07; O, 20.14.

(3,8-Dibromo-9-hydroxymethyl-1,6-dioxa-5 $\lambda^{4}$-selenaspiro[4.4] nona-3,8-dien-4-yl)methanol (7b)

Yield: $0.20 \mathrm{~g}(91 \%)$; colorless solid; $\mathrm{mp} 138-140{ }^{\circ} \mathrm{C}\left(\mathrm{H}_{2} \mathrm{O}\right)$.

IR (KBr): 3392 (br), 3224, 2914, 1641, 1422, 1284, 1227, 1094 $1022,952,758 \mathrm{~cm}^{-1}$.

${ }^{1} \mathrm{H}$ NMR $\left(300 \mathrm{MHz}, \mathrm{DMSO}-d_{6}\right): \delta=3.39$ (br s, $\left.2 \mathrm{H}\right), 4.29(\mathrm{dd}$, $J=12.7,0.9 \mathrm{~Hz}, 2 \mathrm{H}), 4.46(\mathrm{~d}, J=18.0 \mathrm{~Hz}, 2 \mathrm{H}), 4.68(\mathrm{~d}, J=16.5$ $\mathrm{Hz}, 2 \mathrm{H}), 5.01(\mathrm{dd}, J=16.2,1.5 \mathrm{~Hz}, 2 \mathrm{H})$.

${ }^{13} \mathrm{C}$ NMR (75 MHz, DMSO- $\left.d_{6}\right): \delta=58.3$ (2 C), 77.9 (2 C), $133.8(2$ C), $136.2(2 \mathrm{C})$

HRMS: $m / z[\mathrm{M}+\mathrm{H}]^{+}$calcd for $\mathrm{C}_{8} \mathrm{H}_{11} \mathrm{Br}_{2} \mathrm{O}_{4}{ }^{80} \mathrm{Se}$ : 408.8189; found: 408.8165 .

Anal. Calcd for $\mathrm{C}_{8} \mathrm{H}_{10} \mathrm{Br}_{2} \mathrm{O}_{4} \mathrm{Se}$ : C, 23.50; H, 2.46. Found: C, 23.48; $\mathrm{H}, 2.32$.

\section{Reaction of 2,5-Dimethylhex-3-yne-2,5-diol with $\mathrm{SeCl}_{4}$}

The propargyl diol $(0.284 \mathrm{~g}, 2 \mathrm{mmol})$ was added to a soln of $\mathrm{SeCl}_{4}$ $(0.22 \mathrm{~g}, 1 \mathrm{mmol})$ in anhyd $\mathrm{CHCl}_{3}(12 \mathrm{~mL})$ at $0{ }^{\circ} \mathrm{C}$ under argon, and the mixture was stirred at $0{ }^{\circ} \mathrm{C}$ for 30 min until full disappearance of insoluble $\mathrm{SeCl}_{4}$. EtOAc $(70 \mathrm{~mL})$ was added to the reaction mixture, and the mixture was washed with $10 \%$ aq $\mathrm{NaHCO}_{3}(15 \mathrm{~mL})$. The organic phase was dried $\left(\mathrm{MgSO}_{4}\right)$ and the solvent was removed under reduced pressure to give the crude mixture of compounds $\mathbf{1 0}$ and 11 . The products were separated by column chromatography using EtOAc-hexane (1:4) as eluent.
Bis(4-chloro-2,2,5,5-tetramethyl-2,5-dihydrofuran-3-yl)selane (10)

Yield: $75 \mathrm{mg}$ (19\%); yellow oil; $R_{f}=0.7$ (EtOAc-hexane, 1:4).

${ }^{1} \mathrm{H}$ NMR $\left(300 \mathrm{MHz}, \mathrm{CDCl}_{3}\right)$ : $\delta=1.38(\mathrm{~s}, 12 \mathrm{H}), 1.42(\mathrm{~s}, 12 \mathrm{H})$.

${ }^{13} \mathrm{C}$ NMR $\left(75 \mathrm{MHz}, \mathrm{CDCl}_{3}\right): \delta=27.7\left(\mathrm{CH}_{3}\right), 28.9\left(\mathrm{CH}_{3}\right), 86.3$ $\left(C \mathrm{Me}_{2}\right), 88.7\left({ }^{2} J_{\mathrm{C}, \mathrm{Se}} 37 \mathrm{~Hz}, C \mathrm{Me}_{2}\right), 124.9\left({ }^{1} J_{\mathrm{C}, \mathrm{Se}}=132 \mathrm{~Hz},=\mathrm{CSe}\right)$, $141.8(=\mathrm{CCl})$.

4-Chloro-3-(2-chloropropan-2-yl)-5,5-dimethyl-5H-1,2-oxaselenole 2-oxide (11)

Yield: $53 \mathrm{mg}(18 \%)$; white crystals; $\mathrm{mp} 101-102{ }^{\circ} \mathrm{C} ; R_{f}=0.42$ (EtOAc-hexane, 1:4).

${ }^{1} \mathrm{H}$ NMR (700 MHz, $\left.\mathrm{CDCl}_{3}\right): \delta=1.54(\mathrm{~s}, 3 \mathrm{H}), 1.72(\mathrm{~s}, 3 \mathrm{H}), 2.06$ (s, $3 \mathrm{H}), 2.11(\mathrm{~s}, 3 \mathrm{H})$.

${ }^{13} \mathrm{C}$ NMR $\left(175 \mathrm{MHz}, \mathrm{CDCl}_{3}\right): \delta=28.3\left(\mathrm{CH}_{3}\right), 30.5\left(\mathrm{CH}_{3}\right), 32.0$ $\left(\mathrm{CH}_{3}\right), 33.3\left(\mathrm{CH}_{3}\right), 68.5\left(\mathrm{Me}_{2} \mathrm{CCl}\right), 99.1$ (C-5), 144.0 (C-4), 149.7 $\left({ }^{1} J_{\mathrm{C}, \mathrm{Se}}=129.4 \mathrm{~Hz}, \mathrm{C}-3\right)$.

MS (DCI): $m / z(\%)=290.9\left(7.15,[\mathrm{M}+\mathrm{H}]^{+}\right), 255.0(26.3), 223.0$ (18.9), 107.2 (13.2), 85.1 (100).

HRMS: $m / z[\mathrm{M}+\mathrm{H}]^{+}$calcd for $\mathrm{C}_{8} \mathrm{H}_{13} \mathrm{O}_{2}{ }^{35} \mathrm{Cl}_{2}{ }^{80} \mathrm{Se}: 290.9458$; found: 290.9467.

\section{Spiroselenurane 15}

Prepared from ethyl 4-hydroxybut-2-ynoate $(13 ; 170 \mathrm{mg}, 1.35$ $\mathrm{mmol})$ and $\mathrm{SeCl}_{4}(150 \mathrm{mg}, 0.675 \mathrm{mmol})$ following the above procedure for the preparation of $\mathbf{1 0}$ and $\mathbf{1 1}$, with the exception that the mixture was stirred at r.t. for $18 \mathrm{~h}$.

Yield: $8.2 \mathrm{mg}$ (30\%); yellow oil; $R_{f}=0.78$ (hexane-EtOAc, $2: 1$ ).

${ }^{1} \mathrm{H} \mathrm{NMR}\left(\mathrm{CDCl}_{3}, 700 \mathrm{MHz}\right): \delta=1.37(\mathrm{t}, J=7 \mathrm{~Hz}, 6 \mathrm{H}), 4.37(\mathrm{ABq}$ of q, $J=7,11.2 \mathrm{~Hz}, 4 \mathrm{H}), 5.33(\mathrm{~d}, J=17.5 \mathrm{~Hz}, 2 \mathrm{H}), 5.68(\mathrm{~d}$, $J=17.5 \mathrm{~Hz}, 2 \mathrm{H})$.

${ }^{13} \mathrm{C} \mathrm{NMR}\left(\mathrm{CDCl}_{3}, 75 \mathrm{MHz}\right): \delta=14.0\left(\mathrm{CH}_{3}\right), 62.9\left(\mathrm{CH}_{3} \mathrm{CH}_{2}\right), 82.9$ $\left({ }^{2} J_{\mathrm{C}, \mathrm{Se}}=12.4 \mathrm{~Hz}, \mathrm{CH}_{2} \mathrm{O}\right), 138.4\left({ }^{1} J_{\mathrm{C}, \mathrm{Se}}=135.5 \mathrm{~Hz},=\mathrm{CSe}\right), 154.1$ $(=\mathrm{CCl}), 159.1(\mathrm{C}=\mathrm{O})$.

\section{Acknowledgment}

This research was supported by The Israel Science Foundation (grant No 919-05). One of the authors (Y. K.) gratefully acknowledges The Israel Ministry of Science, Technology, and Space for a Ph.D. fellowship.

Supporting Information for this article is available online at http://www.thieme-connect.com/ejournals/toc/synthesis.

\section{References}

(1) Rodell, T. C. Crit. Rev. Oxid. Stress Aging 2003, 2, 1344.

(2) Epp, O.; Ladenstein, R.; Wendel, A. Eur. J. Biochem. 1983, $133,51$.

(3) Parnham, M. J.; Graf, E. Biochem. Pharmacol. 1987, 36, 3095.

(4) Parnham, M. J.; Kindt, S. Biochem. Pharmacol. 1984, 33, 3247.

(5) Wendel, A.; Fausel, M.; Safayhi, H.; Tiegs, G.; Otter, R. Biochem. Pharmacol. 1984, 33, 3241.

(6) Müller, A.; Cadenas, E.; Graf, P.; Sies, H. Biochem. Pharmacol. 1984, 33, 3235.

(7) Back, T. G.; Moussa, Z. J. Am. Chem. Soc. 2002, 124, 12104.

(8) Back, T. G.; Moussa, Z.; Parvez, M. Angew. Chem. Int. Ed. 2004, 43, 1268.

(9) Soriano-Garcia, M. Curr. Med. Chem. 2004, 11, 1657. 
(10) Soda, K.; Tanaka, H.; Esaki, N. Biochemistry of Physiologically Active Selenium Compounds, In The Chemistry of Organic Selenium and Tellurium Compounds; Vol. 2; Patai, S., Ed.; Wiley: New York, 1987, 349-365.

(11) (a) Narajji, C.; Karvekar, M. D.; Das, A. K. Indian J. Pharm. Sci. 2007, 69, 344. (b) Alberto, E. E.; Braga, A. L. Selenium and Tellurium Chemistry; Woollins, J. D.; Laitinen, R., Eds.; Springer: Berlin, 2011, 251-283. (c) Bhuyan, B. J.; Lamani, D. S.; Mugesh, G.; Wirth, T. Handbook of Chalcogen Chemistry: New Perspectives in Sulfur, Selenium and Tellurium, 2nd ed. Devillanova, F. A.; du Mont, W.-W., Eds.; RCS Publishing: Oxford, 2013, 25-46.

(12) (a) Mugesh, G.; du Mont, W.-W.; Sies, H. Chem. Rev. 2001, 101, 2125. (b) Mugesh, G. Curr. Chem. Biol. 2013, 7, 47.

(13) For recent reviews, see: (a) Wirth, T. Organoselenium Chemistry: Synthesis and Reactions; Wiley-VCH: Weinheim, 2011, 448. (b) Patai, S. In The Chemistry of Organic Selenium and Tellurium Compounds; Vol. 3; Rappoport, Z., Ed.; Wiley: New York, 2012, 1582.

(14) Maaninen, A.; Chivers, T.; Parvez, M.; Pietikaeinen, J.; Laitinen, R. S. Inorg. Chem. 1999, 38, 4093.

(15) Braverman, S.; Jana, R.; Cherkinsky, M.; Gottlieb, H. E.; Sprecher, M. Synlett 2007, 2663.

(16) Braverman, S.; Cherkinsky, M.; Jana, R.; Kalendar, Y.; Sprecher, M. J. Phys. Org. Chem. 2010, 23, 1114.

(17) For recent reviews on electrophilic selenium addition to alkenes, see: (a) Santi, C.; Santoro, S. Electrophilic Selenium, In Organoselenium Chemistry: Synthesis and
Reactions; Wirth, T., Ed.; Wiley-VCH: Weinheim, 2011, 351. (b) Freudendahl, D.; Wirth, T. New Selenium Electrophiles and Their Reactivity, In Selenium and Tellurium Chemistry; Woollins, J. D.; Laitinen, R., Eds.; Springer: Berlin, 2011, 41-55. For examples of electrophilic selenium anti-addition to alkynes, see: (c) Schmid, G. H.; Garratt, D. G. Chem. Scr. 1976, 10, 76. (d) Saluzzo, C.; Alvernhe, G.; Anker, D.; Haufe, G. Tetrahedron Lett. 1990, 31, 2127. (e) Brunetti, T.; Diddoro, M.; Di Vona, M. L.; Floris, B.; Galloni, P.; Licoccia, S. Eur. J. Org. Chem. 2004, 521. (f) Usuki, Y.; Iwaoka, M.; Tomoda, S. Chem. Lett. $1992,1507$.

(18) Crystallographic data for compounds $\mathbf{7 a}$ and $\mathbf{1 2}$ have been deposited with the Cambridge Crystallographic Data Centre as supplementary publication numbers CCDC 956314 and CCDC 956315, respectively. These data can be obtained free of charge via www.ccdc.cam.ac.uk/data request/cif, or by emailing data_request@ccdc.cam.ac.uk, or by contacting The Cambridge Crystallographic Data Centre, 12 Union Road, Cambridge CB2 1EZ, UK; fax: +44(1223)336033.

(19) Braverman, S.; Pechenick-Azizi, T.; Gottlieb, H. E.; Sprecher, M. Synthesis 2011, 577.

(20) MacInnes, I.; Walton, J. C. J. Chem. Soc., Perkin Trans. 2 1987, 1077.

(21) Amosova, S. V.; Martynov, A. V.; Mahaeva, N. A.; Belozerova, O. V.; Penzik, M. V.; Albanov, A. I.; Yarosh, O. G.; Voronkov, M. G. J. Organomet. Chem. 2007, 692, 946. 